Article

\title{
Analysis and Correction of the Magnetometer's Position Error in a Cross-Shaped Magnetic Tensor Gradiometer
}

\author{
Youyu Yan ${ }^{(D)}$, Yan Ma * and Jianguo Liu \\ School of Marine Science and Technology, Northwestern Polytechnical University, Xi'an 710072, China; \\ yanyouyu@mail.nwpu.edu.cn (Y.Y.); liujianguo@nwpu.edu.cn (J.L.) \\ * Correspondence: mayan303@nwpu.edu.cn
}

Received: 7 January 2020; Accepted: 25 February 2020; Published: 27 February 2020

\begin{abstract}
When using the technique of magnetic gradient tensor measurements to obtain the position of magnetic objects, calibration of the magnetic tensor gradiometer plays a pivotal role in precisely locating the target, and extensive research has been carried out on this up to now. However, previous studies have always lacked sufficient discussion on the position error of magnetometers in magnetic tensor gradiometers caused by inaccurate installment of magnetometers. In this paper, we analyze and correct this position error based on a magnetic dipole source. The result of the simulation exemplifies that the magnetometer's position error will affect the locating accuracy and, therefore, it is worth correcting this error. The relationship between position error and magnetic gradient tensor components is established, followed by an error correction method based on this relationship. Simulations illustrate that this method can effectively decrease the effect caused by the position error of magnetometers and improve the locating performance with locating error and magnetic moment errors dropping from 2 to $0.2 \mathrm{~m}$ and $6 \times 10^{5} \mathrm{~A} \cdot \mathrm{m}^{2}$ to $5 \times 10^{4} \mathrm{~A} \cdot \mathrm{m}^{2}$, respectively.
\end{abstract}

Keywords: magnetic dipole; magnetic gradient tensor; position error of magnetometers; locating error; error correction

\section{Introduction}

Recently, the magnetic gradient tensor system has been extensively applied in geophysical exploration [1], such as underwater target detection and unexploded ordnance [2], due to the advantages of magnetic gradient tensor measurements, the unstoppable development of technology, and the improvement in various platforms [3]. However, the performance of the tensor system is adversely affected by the measurement error caused by specific factors, which are broadly divided into three aspects [4].

Firstly, an inherent error exists in the magnetic gradient tensor system in obtaining the magnetic gradient tensor components, as the magnetic gradient tensor is generally approximated by the numerical difference between two separated magnetometers instead of the differential. As a result, magnetic objects' localization by magnetic field gradient tensor measurement always involves an inherent locating error. This locating error can be corrected by iterations based on the systematic position error distribution patterns [5]. Besides, the geometric configuration of the system will also affect the accuracy of the obtained data. Rong et al. used the hybrid model of an ellipsoid and magnetic dipole array to simulate and analyze the measurement performance of several typical magnetic gradient tensor systems with different geometric configurations, and proposed that the cross-shaped magnetic gradient tensor system has the characteristics of an optimal structure with minimum measurement error [6].

Secondly, systematic errors (scale factors, non-orthogonally, and bias error) of magnetometers due to the limited production process and processing level also cause the inaccuracy in measurements $[7,8]$. 
However, several theories on the mechanism of this error and its corresponding error calibration methods have been proposed and can thus be efficiently solved. In 2001, Gebre proposed a nonlinear, two-step estimation algorithm to calibrate a single solid-state magnetometer, making the magnetometer calibration achievable without rotating the loaded magnetometer platform [9]. Topaz et al. [10-12] analyzed the measurement errors caused by the magnetometer in detail and established the mathematical error model, basically fitting the real situation. Besides, calibration methods based on the functional link artificial neural network (FLANN) were also proposed. In 2019, YaXin et al. [13] proposed a novel calibration method based on tensor invariants in the nonuniform magnetic field without the extra device. The calibration parameters were estimated using the concept of the magnetic gradient tensor and corresponding rotational invariants combined with Levenberg-Marquardt optimization. As many effective and practical calibration methods for the magnetometer's systematic error are present, we do not discuss these topics in this paper.

One of the important and not very well analyzed aspects of error is the inaccurate installment of magnetometers. Magnetometers may rotate slightly along with the installation center point, inevitably causing misalignment errors between magnetic sensors. Even a minor error can multiply serious errors in the measurement accuracy of the tensor system [14]. Mutual calibration between sensors is necessary before using obtained data for location and detection.

Researchers have proposed many fast and efficient calibration methods for misalignment errors until now. Chi et al. obtained the calibration matrix [15] by solving the orthogonal Procrustes problem; Qingzhu et al. proposed that calibration of misalignment errors can be completed just by three sets of measurement data with the same rotation period [16]. All these methods only consider the misalignment error when installing the sensor, but do not consider the position error of magnetometers caused by inaccurate installment. The position of the sensor's center may not accurately be at its geometric center, or magnetometers may not be correctly installed at the required position, especially for the magnetic gradient tensor system with flexible baseline length in which magnetometers will be slightly inaccurately installed along its axis after each change in baseline. This error will lead to a difference between the actual baseline length and standard baseline length of the tensor system, which significantly affects the accuracy of the obtained magnetic gradient tensor and locating results.

Herein, we analyze the magnetometer's position error in depth and simulate how this error influences the locating results. The mathematical relationship between the magnetic gradient tensor components and magnetometer's error is derived. According to the established mathematical model of the magnetometer's position error, the calibration method is proposed, and its feasibility is verified by simulation at the end of the paper.

The paper is structured as follows. First, the theory of the magnetic gradient tensor and location based on the magnetic gradient tensor is introduced. Afterward, the magnetometer's position error and its influence on the location results are analyzed followed by a correction method for it. Finally, the simulation results of the above content are clearly shown.

\section{Magnetic Gradient Tensor and Location Theory}

When discussing the location of the magnetic target, the distance to the target is usually 3 times larger than the physical dimensions of the target, so the target can be regarded as a magnetic dipole source [17]. The magnitude of the magnetic field generated by a magnetic dipole is expressed as follows:

$$
\mathbf{B}=\frac{\mu_{0}}{4 \pi}\left(\frac{3(\mathbf{M} \bullet \mathbf{r}) \mathbf{r}}{r^{5}}-\frac{\mathbf{M}}{r^{3}}\right) .
$$

where $r=|\mathbf{r}|, \mu_{0} \approx 4 \pi \times 10^{-7} \mathrm{H} / \mathrm{m}, \mathbf{M}=\left(M_{x}, M_{y}, M_{z}\right)$, and $\mathbf{r}$ represents the position vector. $\mu_{0}$ is the permeability of vacuum and $\mathbf{M}$ denotes the magnetic moment vector. 
The magnetic gradient tensor $\mathbf{G}$ is defined as the vector gradient of the magnetic field vector $\mathbf{B}$ [3]. It is formally presented in Equation (2).

$$
\mathbf{G}=\nabla \mathbf{B}=\left[\begin{array}{lll}
G_{x x} & G_{x y} & G_{x z} \\
G_{y x} & G_{y y} & G_{y z} \\
G_{z x} & G_{z y} & G_{z z}
\end{array}\right]
$$

Equation (2) shows that the magnetic gradient tensor is symmetrical, and the sum of the three components of the principal diagonal is equal to 0 , so only five of the nine components of the magnetic gradient tensor are independent [18], and it can be expressed by [19]

$$
G_{i j}=\frac{\mu_{0}}{4 \pi}\left[-\frac{15(\mathbf{M} \bullet \mathbf{r}) i j}{r^{7}}+\frac{3\left(M_{i} j+M_{j} i+(\mathbf{M} \bullet \mathbf{r}) \delta_{i j}\right)}{r^{5}}\right] .
$$

$i, j$ represent $x, y, z$, and $\delta_{i j}=1$ for $i=j$ and $\delta_{i j}=0$ for $i \neq j$.

The magnetic gradient tensor data can be measured by using a magnetic sensor array with a certain structure, such as a cross-shaped array, cube-shaped array, and three-dimensional cross-shaped array, to measure the magnitude of the magnetic field along the three orthogonal axes and calculate the difference in each direction. A schematic of the magnetic tensor gradiometer system is depicted in Figure 1. The formula of the cross-magnetic gradient tensor is shown as [20]

$$
\mathbf{G}=\left[\begin{array}{ccc}
\frac{B_{1 x}-B_{3 x}}{2 h} & \frac{B_{1 y}-B_{3 y}}{2 h} & \frac{B_{1 z}-B_{3 z}}{2 h} \\
\frac{B_{2 x}-B_{4 x}}{2 h} & \frac{B_{2 y}-B_{4 y}}{2 h} & \frac{B_{2 z}-B_{4 z}}{2 h} \\
\frac{B_{1 z}-B_{3 z}}{2 h} & \frac{B_{2 z}-B_{4 z}}{2 h} & -\frac{B_{1 x}-B_{3 x}}{2 h}-\frac{B_{2 y}-B_{4 y}}{2 h}
\end{array}\right]
$$

$B_{i j}(i=1,2,3,4 ; j=x, y, z)$ is the output of the magnetometer $s i$, and $h$ is the baseline length of the magnetic tensor gradiometer.

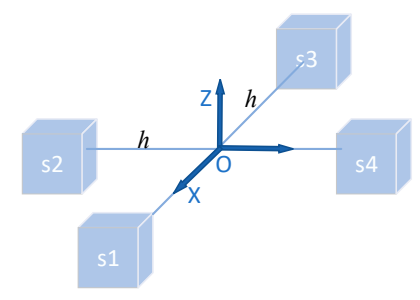

Figure 1. The schematic of the magnetic tensor gradiometer system.

Euler deconvolution is a powerful technique for locating the target, which can be seen as a magnetic dipolar source, as we can directly estimate the target location from the raw data, and any initial estimation of parameters is not required in this method. The expression of the Euler deconvolution algorithm is [21]

$$
\mathbf{r}=-3 \mathbf{G}^{-1} \mathbf{B}
$$

We can locate the magnetic object exactly if we obtain the accurate magnetic gradient tensor components and the magnitude of the magnetic field of the target. The magnetic moment vector can also be estimated correspondingly once we obtain the location of the target [5].

$$
\left[\begin{array}{c}
M_{x} \\
M_{y} \\
M_{z}
\end{array}\right]=2 \pi r\left[\begin{array}{ccc}
3 x^{2}-2 r^{2} & 3 x y & 3 x z \\
3 x y & 3 y^{2}-2 r^{2} & 3 y z \\
3 x z & 3 y z & 3 z^{2}-2 r^{2}
\end{array}\right]\left[\begin{array}{c}
B_{x} \\
B_{y} \\
B_{z}
\end{array}\right]
$$




\section{The Analysis and Correction of Magnetometer's Position Error}

\subsection{Analysis of Locating Error}

In this paper, we analyze the influence of the magnetometer's position error on localization accuracy based on the Euler deconvolution algorithm mentioned above.

The magnetic gradient tensor measurement system constructed by four three-axis fluxgate sensors is located at the measuring point. Here, we assume that all the magnetometers are the standard sensors without any systematic errors, and there is no misalignment error among the magnetometer array. A target that can be modeled as a magnetic dipole is located at the origin of the coordinates. It is assumed that there exists a position error only in the magnetometer $s 1$ when installing the sensor on the cross bracket (Figure 2). The accuracy of $G_{x x}, G_{x y}, G_{x z}$ obtained by this system suffers. According to Equations (1) and (4), these three tensor components, which are acquired by this magnetic tensor gradiometer with the position error of the sensor $s 1$, can be expressed as follows:

$$
\left[\begin{array}{l}
G_{x x^{\prime}} \\
G_{x y^{\prime}} \\
G_{x z^{\prime}}
\end{array}\right]=\frac{1}{2 h} \frac{\mu_{0}}{4 \pi r^{7}}\left[\begin{array}{ccc}
\left(-15 x^{2}+9 r^{2}\right) Q & 3 y r^{2}(2 h+\Delta h)-15 y x Q & 3 z r^{2}(2 h+\Delta h)-15 z x Q \\
3 y r^{2}(2 h+\Delta h)-15 x y Q & \left(-15 y^{2}+3 r^{2}\right) Q & -15 z y Q \\
3 z r^{2}(2 h+\Delta h)-15 x z Q & -15 y z Q & \left(-15 z^{2}+3 r^{2}\right) Q
\end{array}\right]\left[\begin{array}{c}
M_{x} \\
M_{y} \\
M_{z}
\end{array}\right]
$$

where $Q=2 h x+h \Delta h+x \Delta h . G_{x x}{ }^{\prime}, G_{x y}{ }^{\prime}$ and $G_{x z}{ }^{\prime}$ denote incorrect tensor components. $\Delta h$ represents the position error of the magnetometer s1. The specific derivation of Equation (7) is given in Appendix A.

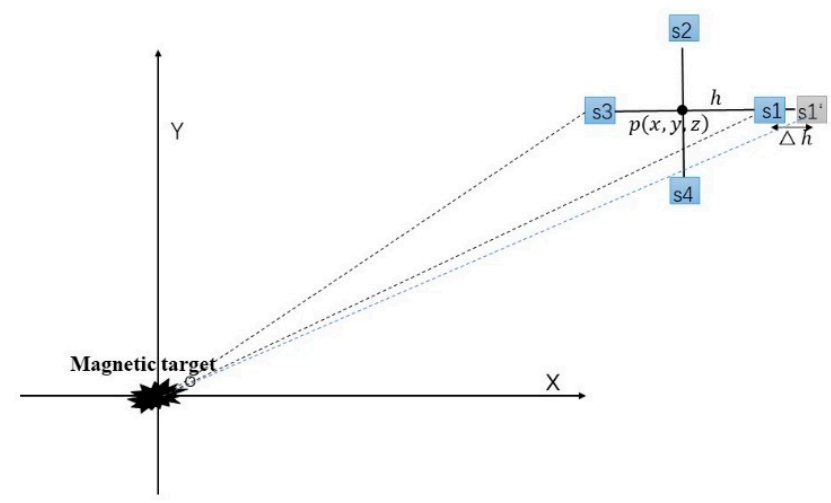

Figure 2. Overhead view of the experiment scene.

The position error of the magnetometer $s 1$ causes errors in the measured magnetic gradient tensor components, which lead to the inaccuracy in the locating results. Equation (5) can be written in a scalar form and, according to the total differential theory, location errors of the target along the three axes can be written as follows:

$$
\begin{aligned}
& \Delta x=\frac{\partial x}{\partial G_{x x}} \Delta G_{x x}+\frac{\partial x}{\partial G_{x y}} \Delta G_{x y}+\frac{\partial x}{\partial G_{x z}} \Delta G_{x z} . \\
& \Delta y=\frac{\partial y}{\partial G_{x x}} \Delta G_{x x}+\frac{\partial y}{\partial G_{x y}} \Delta G_{x y}+\frac{\partial y}{\partial G_{x z}} \Delta G_{x z} . \\
& \Delta z=\frac{\partial z}{\partial G_{x x}} \Delta G_{x x}+\frac{\partial z}{\partial G_{x y}} \Delta G_{x y}+\frac{\partial z}{\partial G_{x z}} \Delta G_{x z} .
\end{aligned}
$$

According to Equation (7), we obtain the expression of $\Delta \mathrm{G}_{x i}$ as

$$
\Delta G_{x i}=G_{x i}-G_{x i}{ }^{\prime} \approx \frac{G_{x i}}{2 h} \Delta h+\frac{\mu_{0}}{8 \pi}\left(\frac{3 M_{x}+3 \delta_{i x} M_{x}}{r^{5}}-\frac{15\left(M_{x} x+M_{y} y+M_{z} z\right) i}{r^{7}}\right) \Delta h .
$$


It is known from Equation (11) that the magnitude of the magnetic gradient tensor error is mainly determined by the first part $\frac{G_{x i}}{2 h} \Delta h$, and $\Delta G_{x i}$ will be larger when the magnetic gradient tensor components are larger or the baseline distance is smaller.

\subsection{The Correction Method for Magnetometer's Position Error}

In summary, the relationship between the measured tensor data with the magnetometer's position error is established as $G_{i j}{ }^{\prime}=f\left(x, y, z, M_{x}, M_{y}, M_{z}, h, \Delta h\right)$.

We can rotate the magnetic tensor gradiometer around the Z-axis to obtain $M(M \geq 2)$ groups of measured magnetic gradient tensor data:

$$
N=\left[\begin{array}{llllll}
N_{x x \theta 1} & N_{x x \theta 2} & N_{x x \theta 3} & \cdots & N_{x x \theta_{(M-1)}} & N_{x x \theta_{M}} \\
N_{x y \theta 1} & N_{x y \theta 2} & N_{x y \theta 3} & \cdots & N_{x y \theta_{(M-1)}} & N_{x y \theta_{M}} \\
N_{x z \theta 1} & N_{x z \theta 2} & N_{x z \theta 3} & \cdots & N_{x z \theta_{(M-1)}} & N_{x z \theta_{M}}
\end{array}\right]
$$

The theoretical inaccurate magnetic gradient tensor data after rotation can be calculated according to Equation (7):

Then, we can establish the error matrix between the actual measured tensor data and the calculated ones:

$$
E=\left[\begin{array}{ccc}
N_{x x \theta 1}-G_{x x \theta 1}{ }^{\prime} & N_{x x \theta 2}-G_{x x \theta 2}{ }^{\prime} & \ldots \\
N_{x y \theta 1}-G_{x y \theta 1}{ }^{\prime} & N_{x y \theta 2}-G_{x y \theta 2^{\prime}} & \ldots \\
N_{x z \theta 1}-G_{x z \theta 1}{ }^{\prime} & N_{x z \theta 3}-G_{x y \theta 3} & \ldots
\end{array}\right] .
$$

Finally, the error coefficient $\Delta h$ can be obtained by solving this nonlinear Equation (14) with a suitable method:

$$
F=\min \left\{E^{T} \bullet E\right\}
$$

\section{Numerical Simulation}

\subsection{Influence Assessment}

In order to investigate the influence of the magnetometers' position error on locating, the simulation experiment is conducted as follows.

Simulation setup:

- A magnetic object, which can be seen as a magnetic dipole, is used as a target and placed at the origin of the coordinates. Its magnetic moment is assumed as $M_{x}=4.5 \times 10^{7} \mathrm{~A} \cdot \mathrm{m}^{2}$, $M_{y}=1 \times 10^{5} \mathrm{~A} \cdot \mathrm{m}^{2}$, and $M_{z}=1 \times 10^{6} \mathrm{~A} \cdot \mathrm{m}^{2}$.

- The baseline length of the magnetic gradient tensor system is $0.5 \mathrm{~m}$ and the precision of the magnetometer is $1 \mathrm{pT}$.

- A $600 \mathrm{~m}$ straight survey line containing 100 observation points is designed. It starts from point $(-300,20,100) \mathrm{m}$ to point $(300,20,100) \mathrm{m}$.

A schematic of the simulation experiment is shown in Figure 3.

The values of the magnetic gradient tensor and magnetic field intensity formed by the magnetic target in each observation point are calculated respectively through Equations (4) and (1). The location information of the target in each observation point can be estimated through Equation (5). Then, the magnetic moment of the target can also be obtained by Equation (6). In order to assess the locating performance, the location error and magnetic moment error of the target are defined as

$$
\begin{aligned}
& \text { err } r_{\text {location }}=\mathbf{r}_{\text {true }}-\mathbf{r}_{\text {estimated }} \text {. } \\
& \text { err } r_{\text {magnetic moment }}=\mathbf{M}_{\text {true }}-\mathbf{M}_{\text {estimated }} \text {. }
\end{aligned}
$$


The location error and magnetic moment error of the target along the survey line are calculated and are shown in Figure 4a. The target locating based on Euler deconvolution has a high accuracy with low location error (less than $0.2 \mathrm{~m}$ ) and comparatively low magnetic moment error (less than $5 \times 10^{4} \mathrm{~A} \cdot \mathrm{m}^{2}$ ), which can be seen in Figure $4 \mathrm{a}$.

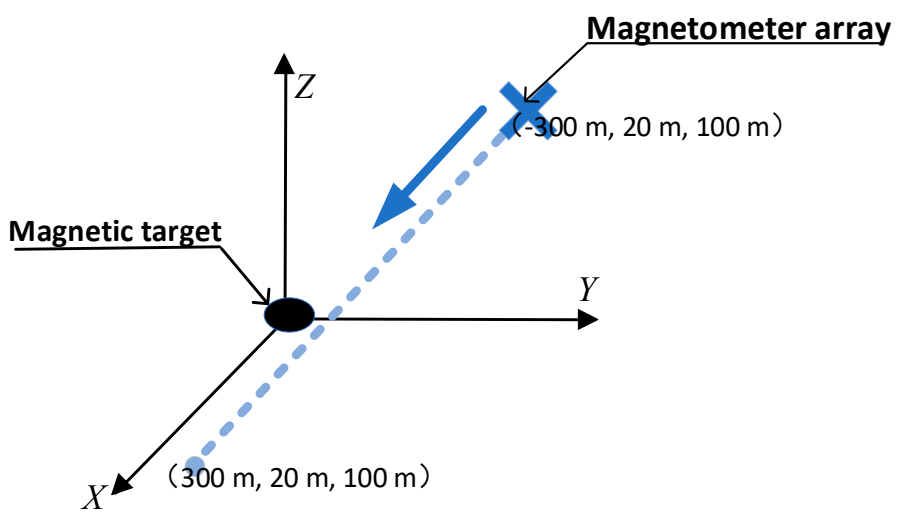

Figure 3. The schematic of the simulation experiment.

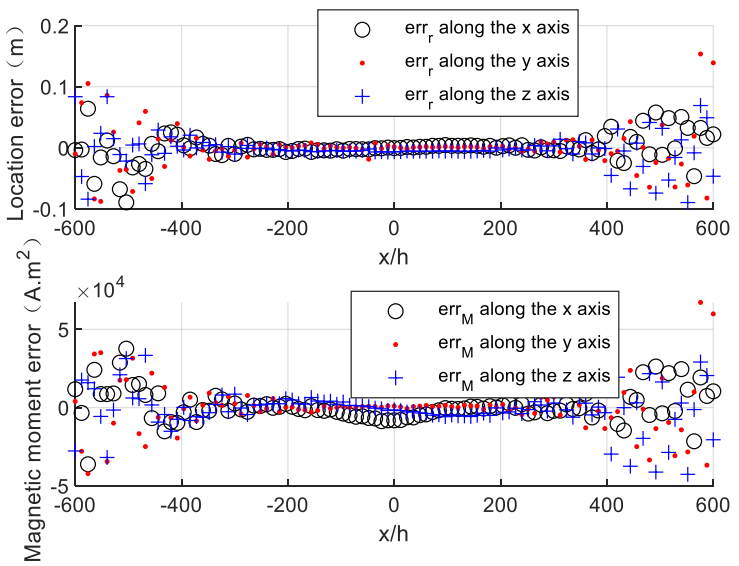

(a)

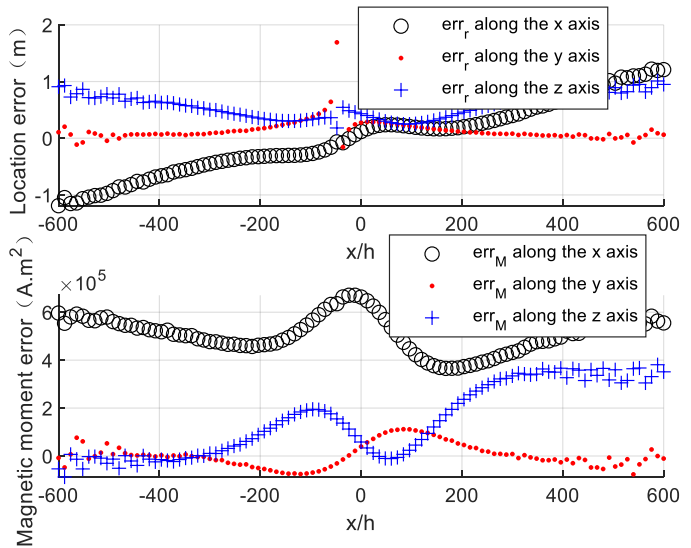

(b)

Figure 4. Locating results by using Euler deconvolution. (a) Locating without position error. (b) Locating with position error.

The position error of the magnetometer s1 $(\Delta h=0.005 \mathrm{~m}, \Delta h / 2 h=0.5 \%)$ is added in the magnetic tensor gradiometer and the simulation experiment is carried out again. The simulation result is shown in Figure $4 \mathrm{~b}$. The locating accuracy seriously degrades compared to Figure $4 \mathrm{a}$. The location error and magnetic moment error of the target increase to $2 \mathrm{~m}$ and $6 \times 10^{5} \mathrm{~A} \cdot \mathrm{m}^{2}$, respectively, due to the presence of the magnetometer's position error.

We can calculate the location error of the target caused by the position error of the magnetometer $s 1$ in each observation point through Equations (8), (9), and (10). The result is shown in Figure 5a, which is nearly the same as the experimental results in Figure $4 \mathrm{~b}$. The magnetic gradient tensor and magnitude of the gradient tensor error along the survey line are calculated through Equation (4) and Equation (11) and are shown in Figure 5b,c. The differentiation of the target's position vector to the magnetic gradient tensor is drawn and shown in Figure 5e. 


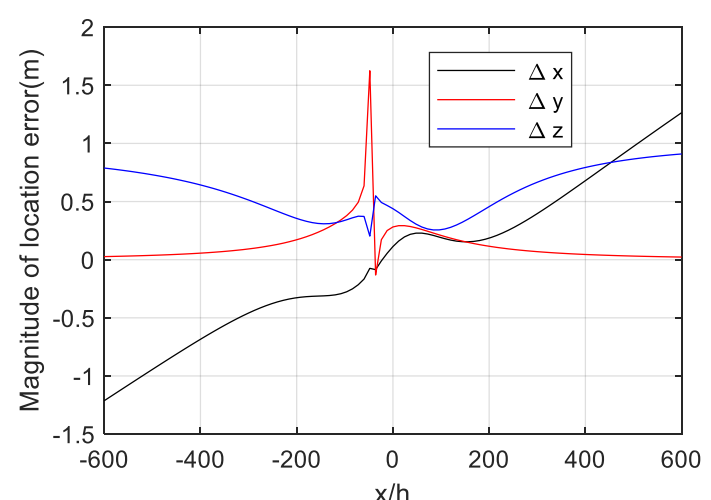

(a)

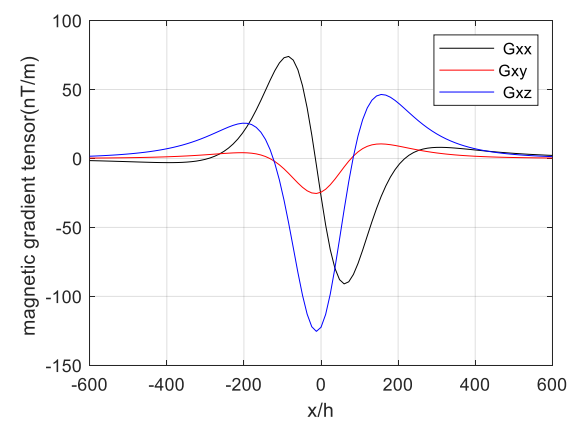

(c)

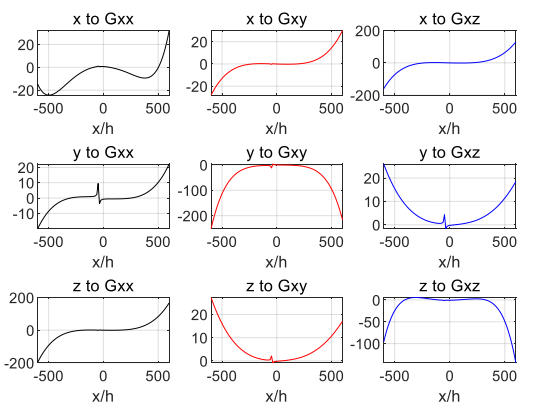

(e)

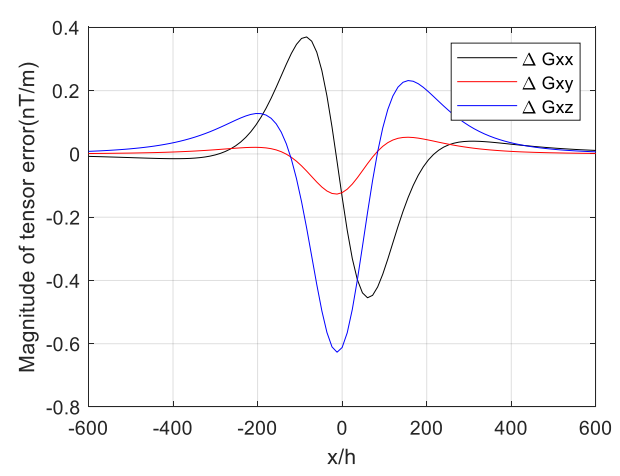

(b)

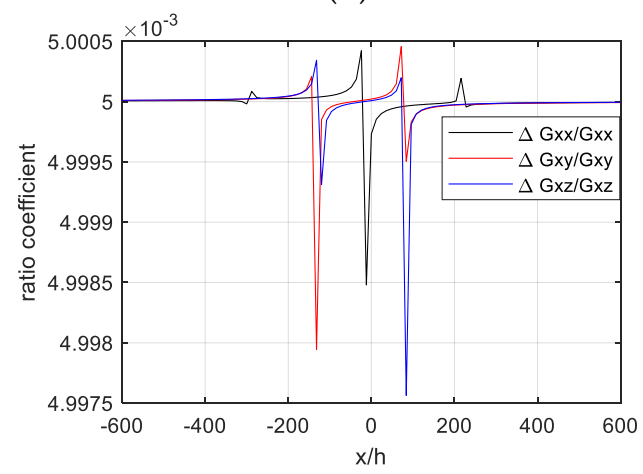

(d)

Figure 5. Influence analysis. (a) The magnitude of location error, (b) the magnitude of tensor error, (c) the magnetic gradient tensor, (d) ratio coefficient between gradient tensor error and gradient tensor, and (e) differential of position vector to magnetic gradient tensor.

The tendency of the gradient tensor error is the same as that of the magnetic gradient tensor according to Figure $5 b, c$, and the ratio coefficient between them is almost equal to $\Delta h / 2 h=0.5 \%$, which can be seen in Figure $5 \mathrm{~d}$. This means that the magnitude of the magnetic gradient tensor error caused by the magnetometer's position error is mainly determined by the first part $\frac{G_{x i}}{2 h} \Delta h$ in Equation (11). Thus, the formula of $\Delta G_{x i}$ can be simplified as follows:

$$
\Delta G_{x i}=G_{x i}-G_{x i}{ }^{\prime} \approx \frac{G_{x i}}{2 h} \Delta h .
$$

As shown in Figure 5e, we can see the tendency of the differential variation of location vectors $x, y$, and $z$ to $G_{x x}, G_{x y}$ and $G_{x z}$ respectively. Their values are almost zero in the near-target area, while they sharply surge in the far target area. The location errors of the target in Figure $5 \mathrm{a}$ is determined by the differential of location vectors to the magnetic gradient tensor and the magnetic gradient tensor error. 


\subsection{Error Correction}

In this section, a simulation experiment is designed to test the proposed correction method. Simulation setup:

- A magnetic object with known magnetic moment $\left(M_{x}=4.5 \times 10^{7} \mathrm{~A} \cdot \mathrm{m}^{2}, M_{y}=1 \times 10^{5} \mathrm{~A} \cdot \mathrm{m}^{2}\right.$, and $\left.M_{z}=1 \times 10^{6} \mathrm{~A} \cdot \mathrm{m}^{2}\right)$ is placed at the origin of the coordinates.

- $\quad$ Preset $\Delta h=0.001 \mathrm{~m}, \Delta h=0.003 \mathrm{~m}, \ldots \ldots, \Delta h=0.01 \mathrm{~m}$.

- The baseline length of the magnetometer array is $0.5 \mathrm{~m}$ and the precision of the magnetometer is 1 pT. The measurement points, which are used to estimate $\Delta h$, can be selected arbitrarily. Herein, two points, $(20,20,100)$ and $(-300,20,100) \mathrm{m}$, with the same $\mathrm{Y}$ and $\mathrm{Z}$ of the survey line in Section 4.1 , are selected to exemplify the correction method.

The magnetometer array is rotated every $30^{\circ}$ around the Z-axis to obtain more than 6 sets of measured magnetic gradient tensor values. The unconstrained nonlinear equation is established according to Equation (14) and the error parameters are estimated. The solution error of $\Delta h$ is defined as

$$
\operatorname{err}_{\Delta h}=\left|\Delta h_{\text {preset }}-\Delta h_{\text {estimated }}\right|
$$

The simulation results are listed in Table 1.

Table 1. The preset and estimated parameters in the simulation.

\begin{tabular}{cccccc}
\hline \multirow{2}{*}{ Number } & Preset $\Delta \boldsymbol{h}(\mathbf{m})$ & \multicolumn{2}{c}{$(\mathbf{2 0 , 2 0 , 1 0 0 )} \mathbf{~}$} & \multicolumn{2}{c}{$(\mathbf{- 3 0 0 , 2 0 , 1 0 0 )} \mathbf{~ m}$} \\
\cline { 3 - 6 } & & Estimated $\Delta \boldsymbol{h}(\mathbf{m})$ & err $_{\Delta \boldsymbol{h}}(\mathbf{m})$ & Estimated $\Delta \boldsymbol{h}(\mathbf{m})$ & err $_{\Delta \boldsymbol{h}}(\mathbf{m})$ \\
\hline 1 & 0.001 & 0.0007 & 0.0003 & 0.001 & 0 \\
2 & 0.002 & 0.0017 & 0.0003 & 0.0019 & 0.0001 \\
3 & 0.003 & 0.0027 & 0.0003 & 0.0029 & 0.0001 \\
4 & 0.004 & 0.0036 & 0.0004 & 0.0039 & 0.0001 \\
5 & 0.005 & 0.0045 & 0.0005 & 0.0049 & 0.0001 \\
6 & 0.007 & 0.0062 & 0.0008 & 0.0068 & 0.0002 \\
7 & 0.008 & 0.0071 & 0.0009 & 0.0077 & 0.0003 \\
8 & 0.01 & 0.0089 & 0.0011 & 0.0096 & 0.0004 \\
\hline
\end{tabular}

As shown in Table 1, the estimated error parameters are basically equal to the theoretical data at these two points. This means that $\Delta h$ can be successfully obtained at both these points and the calibration method proposed in Section 3.2 is feasible. The solution errors of $\Delta h$ at the point $(-300,20,100) \mathrm{m}$ are much smaller, compared to the results at point $(20,20,100) \mathrm{m}$, presumably because the distance between the point $(-300,20,100) \mathrm{m}$ and the magnetic dipole is larger. Equation (7) is deducted based on the assumption that the baseline length of the magnetometer array reaches comparatively close to zero. When the distance between the measurement point and the magnetic dipole is large enough, the baseline length of the magnetometer array can be seen as infinitesimal, and the inaccurate magnetic tensor data calculated by Equation (7) can better fit the real measured data. Therefore, the estimation of $\Delta h$ has a better performance.

We use the estimated result of point $(20,20,100) \mathrm{m}$ to correct the magnetic gradient tensor along the survey line in Section 4.1 according to Equation (17) and use corrected data to carry out the locating experiment again. The locating result after correction is presented in Figure 6. The location errors and the magnetic moment errors of the target both largely declined after error correction. 


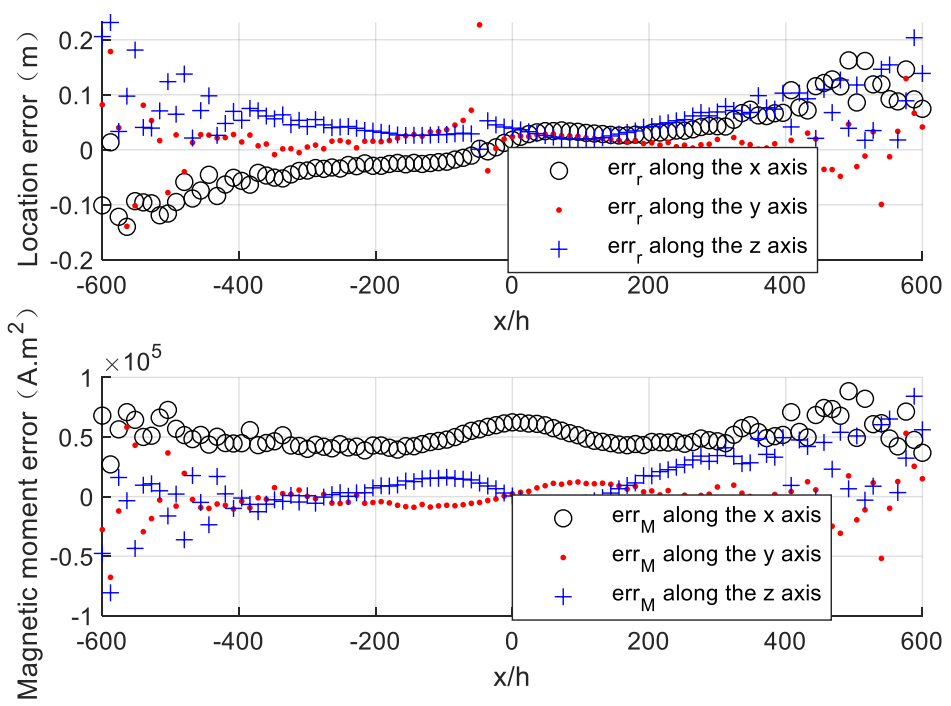

Figure 6. Locating results after calibration.

\section{Conclusions}

The position error of magnetometers in the magnetic gradient tensor system was analyzed based on the model of a magnetic dipole. It can be seen from the simulation results that this error will seriously affect the locating accuracy, so it is highly necessary to analyze and correct such an error for precision. The function between position error and magnetic gradient tensor components is established. An error calibration method based on this function is proposed and this method is profound for further improving the capacity of the tensor system and performance of the localization algorithm.

Author Contributions: Y.Y., Y.M. and J.L. conceived and designed the study; Y.Y. and Y.M. derived the formula; Y.Y., Y.M. and J.L. contributed to data analysis; Y.Y. wrote the paper; Y.Y., Y.M. and J.L. reviewed and edited the manuscript; Y.M. acquired founding. All authors of article provided substantive comments. All authors have read and agreed to the published version of the manuscript.

Funding: National Natural Science Foundation of China: 61901386.

Conflicts of Interest: The authors declare no conflict of interest.

\section{Appendix A}

Derivation of Equation (7):

$$
\begin{array}{r}
B_{1 x^{\prime}}=\frac{\mu_{0}}{4 \pi}\left(\frac{3\left(M_{x}(x+h+\Delta h)+M_{y} y+M_{z} z\right)(x+h+\Delta h)-M_{x}(x+h+\Delta h)^{2}-M_{x} y^{2}+M_{x} z^{2}}{\left((x+h+\Delta h)^{2}+y^{2}+z^{2}\right)^{\frac{5}{2}}}\right) \\
=\frac{\mu_{0}}{4 \pi}(f(x+h+\Delta h) g(x+h+\Delta h)) \\
B_{3 x}=\frac{\mu_{0}}{4 \pi}\left(\frac{3\left(M_{x}(x-h)+M_{y} y+M_{z} z\right)(x-h)-M_{x}(x-h)^{2}-M_{x} y^{2}+M_{x} z^{2}}{\left((x-h)^{2}+y^{2}+z^{2}\right)^{\frac{5}{2}}}\right) \\
=\frac{\mu_{0}}{4 \pi}(f(x-h) g(x-h))
\end{array}
$$

$B_{1 x^{\prime}}$ and $B_{3 x}$ denote the measured magnetic field intensity by senor $s 1$, which has the position error, and sensor $s 3$, respectively. We can write the mathematical expression of $B_{1 x}{ }^{\prime}$ and $B_{3 x}$ according to Equation (1), and $G_{x x}{ }^{\prime}$ can be written as Equation (A3) according to Equation (4).

$$
\begin{aligned}
& G_{x x}{ }^{\prime}= \lim _{2 h \rightarrow 0} \frac{B_{1 x^{\prime}}-B_{3 x}}{2 h}=\frac{\mu_{0}}{4 \pi} \lim _{2 h \rightarrow 0} \frac{(f(x+h+\Delta h) g(x+h+\Delta h)-f(x-h) g(x-h))}{2 h} \\
&=\frac{\mu_{0}}{4 \pi} \lim _{2 h \rightarrow 0} \frac{1}{2 h}(f(x+h+\Delta h) g(x+h+\Delta h)-f(x-h) g(x+h+\Delta h) \\
&-f(x-h) g(x-h)+f(x-h) g(x+h+\Delta h))
\end{aligned}
$$


Simplify Equation (A3):

$$
\begin{aligned}
G_{x x}{ }^{\prime}=\frac{\mu_{0}}{4 \pi} \lim _{2 h \rightarrow 0} \frac{1}{2 h} & \left\{\left(2 M_{x}\left(4 x h+2 h \Delta h+\Delta h^{2}+2 x \Delta h\right)+3 M_{y} y(2 h+\Delta h)+3 M_{z} z(2 h+\Delta h)\right) r^{-5}\right. \\
& -\frac{5}{2} A \bullet\left(4 h x+\Delta h^{2}+2 h \Delta h+2 x \Delta h\right) r^{-7} \\
& \left.-A \bullet \frac{35}{8}\left(\left(2 h x+h^{2}+\Delta h^{2}+2 h \Delta h+2 x \Delta h\right)^{2}-\left(-2 h x+h^{2}\right)\right) r^{-9}+\cdots\right\}
\end{aligned}
$$

where $A=3\left(M_{x} x+M_{y} y+M_{z} z\right) x-M_{x} x^{2}-M_{x} y^{2}+M_{x} z^{2}$.

When $2 h$ reaches extremely close to zero, Equation (A4) can be simplified as

$$
\begin{aligned}
& G_{x x}{ }^{\prime} \approx \frac{\mu_{0}}{4 \pi} \frac{1}{2 h}\left\{\left(2 M_{x}(4 x h+2 h \Delta h+2 x \Delta h)+3 M_{y} y(2 h+\Delta h)+3 M_{z} z(2 h+\Delta h)\right) r^{-5}\right. \\
& \left.+\left(3\left(M_{x} x+M_{y} y+M_{z} z\right) x-M_{x} x^{2}-M_{x} y^{2}-M_{x} z^{2}\right)\left(-\frac{5}{2}(4 h x+2 h \Delta h+2 x \Delta h) r^{-7}\right)\right\}
\end{aligned}
$$

The mathematical expression of $G_{x y}{ }^{\prime}$ and $G_{x z}{ }^{\prime}$ can be obtained in the same way. In order to test the fitting degree of measured data and data calculated by the mathematical method, the simulation is carried out and the result is shown in Figure A1. The calculated data fit the measured data well when $\Delta h$ is small relative to $h$ according to the simulation result.

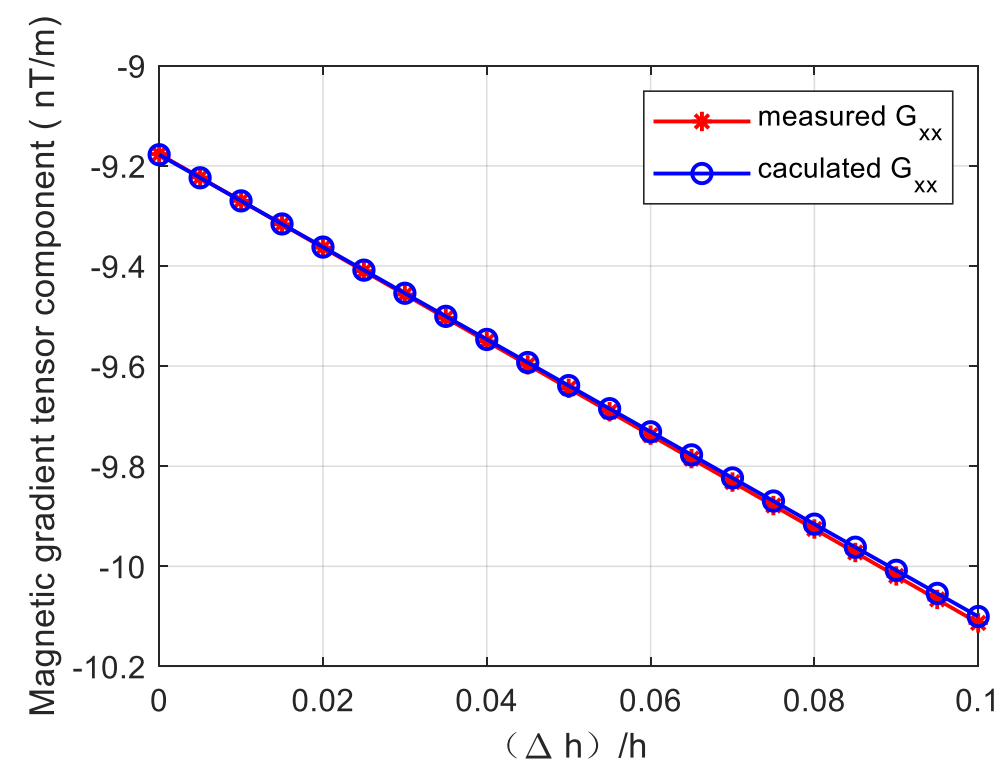

Figure A1. The simulation results of fitting degree.

The simulation conditions are listed as follows:

- The magnetic moment of the magnetic object: $M_{x}=4.5 \times 10^{7} \mathrm{~A} \cdot \mathrm{m}^{2}, M_{y}=1 \times 10^{5} \mathrm{~A} \cdot \mathrm{m}^{2}$, and $M_{z}=1 \times 10^{6} \mathrm{~A} \cdot \mathrm{m}^{2}$.

- $h=0.2 \mathrm{~m}$ and $0 \leq \frac{\Delta h}{h} \leq 0.1$.

- The observation point is arbitrarily selected as $(20,10,10) \mathrm{m}$.

\section{References}

1. Nabighian, M.N.; Grauch, V.J.S.; Hansen, R.O.; Lafehr, T.R.; Phillips, J.D.J.G. The historical development of the magnetic method in exploration. Geophysics 2005, 70, 33ND. [CrossRef]

2. $\mathrm{Mu}, \mathrm{Y}$; Zhang, $\mathrm{X}$.; Xie, W. Calibration of Magnetometer Arrays in Magnetic Field Gradients. IEEE Magn. Lett. 2019, 10, 1-5. [CrossRef]

3. Clark, D.A. New methods for interpretation of magnetic vector and gradient tensor data I: Eigenvector analysis and the normalised source strength. Explor. Geophys. 2018, 45, 267-282. [CrossRef]

4. Qingzhu, L.; Zhining, L.; Yingtang, Z.; Gang, Y.; Hong-bo, F. Research Progress of Magnetic Gradient Tensor System and its Error Calibration. J. Acad. Armored Force Eng. 2017, 31, 72-81. [CrossRef] 
5. Sansheng, W.; Mingji, Z.; Ning, Z.; Qiang, G. Calculation and correction of magnetic object positioning error caused by magnetic field gradient tensor measurement. Syst. Eng. Electron. 2018, 29, 456-461. [CrossRef]

6. Cheng, C.; Jun-wei, L. Comparative Analysis of Magnetic Gradient Tensor System Structure. Command. Control Simul. 2019, 41, 46-49. [CrossRef]

7. Merayo, J.M.G.; Brauer, P.; Primdahl, F.; Petersen, J.R.; Nielsen, O.V. Scalar calibration of vector magnetometers. Meas. Sci. Technol. 2000, 11, 120-132. [CrossRef]

8. Ousaloo, H.S.; Sharifi, G.; Mahdian, J.; Nodeh, M.T. Complete Calibration of Three-Axis Strapdown Magnetometer in Mounting Frame. IEEE Sens. J. 2017, 17, 7886-7893. [CrossRef]

9. Gebre-Egziabher, D.; Elkaim, G.H.; Powell, J.D.; Parkinson, B.W. A Non-Linear, Two-Step Estimation Algorithm for Calibrating Solid-State Strapdown Magnetometers. In Proceedings of the International St Petersburg Conference on Navigation Systems, Saint Petersburg, Russia, 28-30 May 2001.

10. Xiang, G.; Shenggang, Y.; Bin, L. Study on the steering differential calibration method for magnetic gradiometer base on tri-axis fluxgate. Chin. J. Sci. Instrum. 2016, 37. [CrossRef]

11. Yu, H.; Yanling, H. Correction of fluxgate field gradiometer based on FLANN. J. Huazhong Univ. Sci. Tech. (Nat. Sci. Ed.) 2011, 39, 83-87. [CrossRef]

12. Yu, H.; Yanling, H. Error correction of magnetic field component gradiometer based on FLANN and least-squares. Chin. J. Sci. Instrum. 2012, 33, 911-917. [CrossRef]

13. Mu, Y.; Wang, C.; Zhang, X.; Xie, W. A Novel Calibration Method for Magnetometer Array in Nonuniform Background Field. IEEE Trans. Instrum. Meas. 2019, 68, 3677-3685. [CrossRef]

14. Xiang, L.; Yongjun, W.; Zhi, L. Inter-Triad Misalignment of Vector Field Sensors in Attitude and Heading Reference Systems and Its Calibration. Chin. J. Sens. Actuators 2017, 30, 266-271. [CrossRef]

15. Chi, C.; Lv, J.W.; Wang, D.; Yu, Z.T.; Huang, J.L. Integrated Calibration for the Cross Magnetic Gradiometer. J. Magn. 2017, 22, 483-490. [CrossRef]

16. Li, Q.-Z.; Li, Z.-N.; Zhang, Y.-T.; Yin, G. Fast rotation calibration of sensor array in magnetic gradient tensor system. Opt. Precis. Eng. 2018, 26, 1813-1826. [CrossRef]

17. Wiegert, R.; Oeschger, J. Generalized magnetic gradient contraction based method for detection, localization and discrimination of underwater mines and unexploded ordnance. In Proceedings of the OCEANS 2005 MTS/IEEE, Washington, DC, USA, 17-23 September 2005; Volume 1322, pp. 1325-1332. [CrossRef]

18. Gang, Y.; Yingtang, Z.; Zhining, L.; Hongbo, F.; Guoquan, R. Detection of ferromagnetic target based on mobile magnetic gradient tensor system. J. Magn. Magn. Mater. 2016, 402, 1-7. [CrossRef]

19. Wiegert, R.; Lee, K.; Oeschger, J. Improved magnetic STAR methods for real-time, point-by-point localization of unexploded ordnance and buried mines. In Proceedings of the OCEANS 2008, Quebec, QC, Canada, 15-18 September 2008; pp. 1-7. [CrossRef]

20. Gang, Y.; Yingtang, Z.; Hongbo, F.; Guang, Z.; Guoquan, R. Linear calibration method of magnetic gradient tensor system. Measurement 2014, 56, 8-18. [CrossRef]

21. Nara, T.; Suzuki, S.; Ando, S. A Closed-Form Formula for Magnetic Dipole Localization by Measurement of Its Magnetic Field and Spatial Gradients. IEEE Trans. Magn. 2006, 42, 3291-3293. [CrossRef]

(C) 2020 by the authors. Licensee MDPI, Basel, Switzerland. This article is an open access article distributed under the terms and conditions of the Creative Commons Attribution (CC BY) license (http://creativecommons.org/licenses/by/4.0/). 